\title{
Hemophilia: A High Cost Low Volume Disease: Suitable Preventive Strategies for Developing and Developed Countries
}

\author{
Kanjaksha Ghosh*, ${ }^{*}$, Shrimati Shetty ${ }^{1}$ and Kinjalka Ghosh ${ }^{2}$

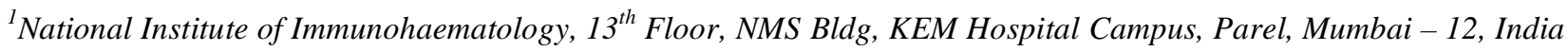 \\ ${ }^{2}$ Department of Community medicine, Pravara Institute of Medical Sciences, Loni, Ahmednagar, Maharashtra, India
}

\begin{abstract}
Hemophilia A \& B are congenital bleeding disorders affecting 1:10-20,000 population and 1:20 to 40,000 population respectively. Hemophilia represents the prototype of high cost low volume disease. Eighty persons of world hemophilia population lives in financially poor developing countries, where < $2 \%$ of GDP is usually spent for total health care. In India, with a population of more than 1 billion and growth rate of around $2 \%$ atleast 2000 new hemophilia patients are born every year. On a conservative estimate $50 \%$ of them have severe disease and with modern treatment, will require additional USD 36,000/year/patient i.e a 36 million dollar incremental additional health care burden each year to manage these 1000 severe hemophilia patient. Which is added to our existing pool of haemophiliacs, estimated at 1,00,000$1,20,000$.

One of the ways to manage this challenge is to establish prenatal diagnostic centres, for hemophilia, spread widely for easy accessibility. These diagnostic centers can eventually develop into prenatal diagnosis centres for other diseases like common hemoglobinopathies and they should be equipped with modest molecular diagnostic facilities.

Presently there are a series of techniques of different levels of difficulties and complexities, available for foetal tissue sampling and laboratory detection of prenatal diagnosis of hemophilia, allowing a centre to chose the technology suitable for their expertise and financial capability.
\end{abstract}

Keywords: Prenatal Diagnosis, Carrier Detection, Haemophilia A \& B, Developing Countries, Preventive strategies.

\section{THE CHALLENGE}

Hemophilia A affects 1: 10 - 20,000 pregnancy while hemophilia B affects one in 20 - 40,000 pregnancies worldwide [1]. In developed countries where treatment is either free through national health service or through health insurance schemes and there is high quality health care throughout the country hemophilia patients are looked after well and they can live normally, though always living under the shadow of unusual blood borne pathogens, inhibitor development and ever increasing fear of severe bleed in the brain, which has a life time cumulative incidence rate of $10 \%$ for severe hemophiliacs [2]. Severe hemophilia patients can now live an almost normal life without any disability if they start receiving prophylaxis from $2 \mathrm{yrs}$ of life; requiring $3000-$ 4000 I.U/Kg of factor concentrates per year. Taking an average weight of a child at $30 \mathrm{Kg}$ and price per unit of factor concentrates (Plasma derived) at 30 cents/unit, total cost works out to be USD 36,000/year. This is an extremely high cost proposition for a developing country where per capita yearly income varies between 530-1000 USD/year [3]. Hence hemophilia may be considered as high cost low volume disease. Moreover the classical 80-20 gap applies for hemophilia too i.e $80 \%$ of the world hemophilia receive only $20 \%$ of the resources for management of these patients [4].

A disease which is high cost to manage needs to be prevented. One of the ways to prevent the birth of severe hemo-

*Address correspondence to this author at the National Institute of Immunohaematology, 13 th Fl KEM Hospital, Parel, Mumbai-400012, India; Tel: +91-22-24132928; Fax: +91-22-24138521;

E-mail: kanjakshaghosh@hotmail.com philia patients is by a process of prenatal diagnosis followed by post diagnosis counseling [5].

Prenatal diagnosis may involve various kinds of complex laboratory techniques with different levels of complexities and misdiagnosis rates. In this review we will discuss the various techniques for prenatal diagnosis and find out how these techniques can be optimally blended to suite the purpose in countries with different levels of development.

One of the pre requisites of carrier detection and prenatal diagnosis in hemophilia is the correct diagnosis of the disease. Correct diagnosis of hemophilia A (Factor VIII deficiency) and hemophilia B (Factor IX deficiency) depends on availability of screening coagulation tests (APTT, PT, TT) correction studies and finally one stage APTT based assay of the deficiency factors to asses the severity of the disease along with assessment to rule out Von willebrand disease as a cause of factor VIII deficiency.

In India there are more than 600 districts and each district has an average of 2 million population (Approximately 200 hemophilia A and 50 - 100 hemophilia patients are expected in each district based on the population prevalence). However, evaluation by hemophilia Federation of India show that except in big cities like Delhi, Mumbai, Kolkata there are very few places where dependable coagulation factor assays are available. We have every reason to believe that situations are no better in the adjoining developing countries. On the last count there were only $26-30$ laboratories in the country who could do correction studies and factor assays. 
It is imperative for the health care givers (Government in most of the developing countries) to develop at least an elementary coagulation laboratory in each district if we have to clearly identify the families affected with hemophilia.

What are the impediments in the development of a coagulation laboratory in a district hospital taking care of at least 2 million population?

Developing an elementary coagulation laboratory in the district hospital is not a high cost proposition, moreover this kind of laboratory is extremely useful for managing many other patients in surgery, gynaecology and general medicine in addition to few hundred patients affected with hemophilia in each district . It is the lack of political will (Health related expenditure in most of the developing countries is < $2 \%$ of GDP) which lies at the heart of the problem.

Moreover in many developing countries $60-80 \%$ of the population lives in rural areas where health care facilities are limited and even non existent. However hemophilia, particularly severe hemophilia is such a disease that many such families travel hundreds to kilometer to get the relevant investigations done but finally cannot afford the treatment.

Recently some private laboratories in the country have developed extensive network of sample collection and quick transportation facilities to their testing centers situated in cities. These laboratories have provided alternative ways of good laboratory access for remote population in the country and can be utilized for the purpose of diagnosing haemophilia patients in remote areas.

Once hemophilia is diagnosed in the index case, extensive pedigree construction and analysis of family tree allows one to find out other possible at risk members and carriers for further detection and analysis. The daughters of affected father and a lady who has a hemophilic son and a hemophilic brother or has two hemophilic sons are known as confirmed carriers and they generally don't' need any further testing to determine their carrier status.

There is a need to identify the female carrier of this disease because not only do they need to be counseled for prenatal diagnosis, but also a proportion of them may bleed because of lower levels of deficient factors due to extreme lyonisation [6]. Carrier detection of hemophilia can only be definitely done by using techniques of molecular biology but $10-15 \%$ of carrier females may have deficient factors below $0.3 \mathrm{IU} / \mathrm{ml}$ allowing them to be diagnosed as carriers without molecular testing. Prenatal diagnosis involve two important steps (i) Getting an adequate sample from developing foetus, chorionic villus, amniotic fluid, cord blood. (ii) Testing the collected sample for evidence of disease.

\section{FOETAL TISSUE SAMPLING}

Foetal tissue sampling is an invasive procedure and generates anxiety in the mother. Gynaecologists and sonologists can initially screen the pregnancy for the period of gestation as the desirable period for chorionic villus sampling is 10-14 weeks. The sex of the foetus can also be diagnosed by ultrasound and if it is a female foetus further invasive tests for prenatal diagnosis may not be warranted, as rarely a female is affected with haemophilia. DNA extracted from foetal sample (Chorionic Villus, Aminocentesis) can be tested for sex of the foetus using $\mathrm{Y}$ chromosome $\mathrm{X}$ chromosome specific markers. This sample can also be used to exclude maternal tissue contamination of fetal sample by VNTR or STR marker analysis. Now a days techniques are evolving where fetal sex can be determined from DNA extracted from mothers peripheral blood or serum without any invasive procedure.

It is always preferable to be able to get a tissue sample as early in pregnancy as possible. This reduces complications in the form of foetal loss. Chorionic Villus sampling done very early in pregnancy increases the risk of limb abnormality in the foetus. Hence desirable time for foetal samplying in between $10-14$ weeks of gestation and this procedure has $\mathrm{s}$ low complication - rate of $0.1-1 \%$ foetal loss [7]. Other two procedures i.e Amniocentesis is easier and can be done between 14-18 weeks of gestation. Amniocentesis has a higher complication rate of $1-2 \%$ foetal loss, this complication rate is same as the third technique i.e foetal blood sampling between 18-20 weeks of gestation (Cordocentesis). Foetal samples obtained from all these three procedure needs an initial evaluation and processing either to dissect out maternal tissue in chorionic villus, concentration of foetal cells from amniotic fluid or separation of citrated plasma from cordocentesis preparation. All the three types of samples can be used for molecular biological diagnosis after DNA extraction from the samples thus obtained [8]. Only properly collected citrated cordocentesis sample is suitable for use in phenotypic diagnosis by factor VIII or factor IX assay as the case may be [9]. One of the great assets in developing countries like India is the availability of well trained gynaecologist down to the level of districts and they could easily be trained to do foetal tissue/ blood sampling after a minimum period of training.

\section{ALTERNATIVE SIMPLER PHENOTYPING TECH- NIQUES FOR SECOND TRIMESTER PRENATAL DIAGNOSIS}

Molecular biology laboratories and trained molecular biology technicians/scientists capable of doing relevant tests for prenatal diagnosis are not available or are very scarce in these countries. In India there are only 4-5 centers where regular prenatal diagnosis for hemophilia families are carried out [10-13]. Chorionic villus samples though can be done in early pregnancy with some what lesser amount of foetal complication needs a molecular biology laboratory to give the prenatal diagnosis where as cordocentesis sample can be tested both by molecular biology test as well as by factor VIII or Factor IX assays. A simple HPLC technique from cordocentesis sample for diagnosis of homozygous $\beta$ thalassemia has also been developed in this center. Hence it is possible to develop a good cordocentesis facility at a district level for phenotypic prenatal diagnosis of both haemophilia and thalassemia at 17-20 weeks of gestation. The factor assay with little effort may be made available at district level and in expert hands the results are robust $[9,14)$ only. An ultrasound machine with Doppler probe is available easily, down to the level of a district. Cordocentisis followed by factor assays could be initially utilized at district levels for prenatal diagnosis where molecular technique are not available. 


\section{MOLECULAR TECHNIQUE FOR PRENATAL DI- AGNOSIS}

There are two broad category of molecular techniques which are used for prenatal diagnosis.

\section{A. Indirect RFLP Based Gene Tracking Technique}

In this technique many RFLP (Restriction Fragment Length Polymorphism) markers or STR (Short Trandem Repeat) markers spread through out the introns of factor VIII and IX gene or in the vicinity of its immediate extragenic location are used. The idea is to track the affected or healthy gene (Factor VIII or IX as the case may be) from the carrier mother to the foetus. There are several disadvantages of this technique.

i. This technique needs testing of both the affected and unaffected member of the family. The affected sibling, father or maternal grandfather who have only one $\mathrm{X}$ chromosome identifies the linkage with defective factor VIII or IX gene. If a mother has only one affected son and no other affected member in the family by RFLP studies it cannot be definitely concluded whether the mother is a carrier or not. (25\% of haemophilia arise due to new mutation)

ii. Usefulness of different RFLP or STR markers varies from one population to another [15] depending on the heterozygosity rate of that RFLP. Higher the heterozygosity rate (i.e more possible alleles) higher is the usefulness of that marker. Some of the STR markers may have many alleles and with that marker $85-90 \%$ of the population could be informative [16].

iii. If the RFLP or STR markers is extragenic in location it can produce higher rates of misdiagnosis due to meiotic cross over [17].

iv. In many consanguineous populations, because of the homogeneity of the population a string of RFLP from different exons/introns of the affected gene may not

Healthy Father be informative. Hence cannot be used for prenatal diagnosis.

v. RFLP or STR based diagnosis needs at least one affected relative. In developed countries where good health care allows the severe hemophilic to live longer, such a relative/sib is easily available. But the affected relatives may not be alive when prenatal diagnosis of one of the member of the family is required. Presently we preserve the DNA sample for any hemophilia patient who comes to our center for diagnosis and sometimes we have to use these archieved samples.

\section{B. Direct Diagnosis by Detecting the Gene Pathology}

For severe factor VIII deficiency, inversion of intron 22 [18] and Inversion of intron 1 [19] are two pathologies which disorganizes the gene. Inversion of intron 22 is present between $35-45 \%$ of severe hemophilia A patient and Inversion of intron 1 is present is $1-6 \%$ of such patients. These two pathologies can now be detected using PCR based technology and many centers uses these two investigations as the first test for direct detection of pathology in severe hemophilia A patient. This test directly detects the pathology and it does not have the drawbacks of RFLP based diagnosis. However these tests are more demanding and difficult to standardize for factor VIII genes. In those cases of factor VIII deficiency where inversion of intron 1 or intron 22 is not the pathology a combination of conformation sensitive gel electrophoresis (CSGE) coupled with DNA sequencing of the amplified segment with abnormal migration pattern can directly give the mutation and the pathology in a particular case [20,21] for factor VIII and IX genes.

\section{ALGORITHM OF CARRIER DETECTION AND PRENATAL DIAGNOSIS OF HEMOPHILIA SUIT- ABLE FOR DEVELOPING COUNTRIES}

Carrier detection cannot be done without direct or indirect molecular biology techniques except in a small number

\section{Carrier Mother}
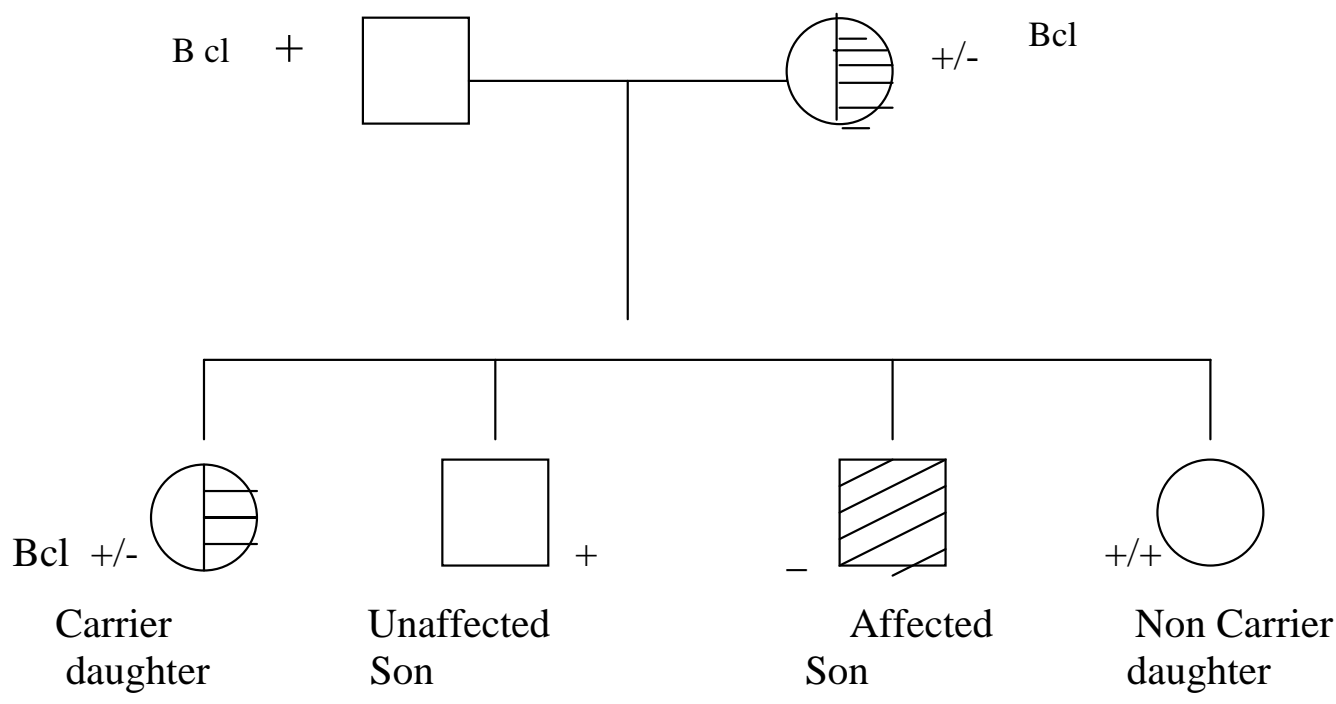

Fig. (1). Showing use of RFLP(Restriction Fragment Length Polymorphism) for carrier/prenatal diagnosis of haemophilia A. 
of carriers with extreme lyonisation where reduced circulating factor level can directly confirm them as carriers.

\section{For molecular biology based test:}

For laboratories who are doing standard molecular biology work. RFLP based diagnosis is easy to adapt. The Fig. (1), shows how RFLP based tracking can tell whether affected gene is present in the foetus (Antenatal Diagnosis) or in the suspected carrier (one normal and one abnormal gene).

3. Laboratories which has higher technical expertise can combine RFLP and STR based investigations with Inversion of intron 22 and intron 1 of factor VIII genes. Combination of these techniques will give some versatility which is not possible for laboratories practicing only RFLP/STR based techniques.

4. The laboratory which has reached still higher levels of expertise can combine all the capabilities of No 3 lab as above with CSGE and DNA sequencing leading to direct detection of mutation in the affected sample. With the development of good Hemophilia registry and birth records these data of the families and family specific mutation can be detected in the extended family members using simpler techniques of abbreviated CSGE with sequencing or just sequencing the affected area of the gene.

5. Laboratories in the developing countries where no molecular biology facility is available can still do prenatal diagnosis by cordocentesis and affected factor assays. This technique will unnecessarily sample female from relatives of haemophilia patients who may not be carriers $(0.1-2 \%$ complications $)$ but the techniques are quite robust and detect cases not detectable by modern tools of genetics [9] and this technique can additionally detect affected foetus for many rare inherited coagulation disorders [22] as well as haemoglobinopathies like $\beta$-thalassemia major, which is often the major genetic problem in the developing countires.

\section{TRAINING MANPOWER AND DEVELOPING LABORATORY FACILITIES IN DEVELOPING COUNTRIES}

One of the major challenges for developing carrier detection and prenatal diagnosis facilities are:

i) Non availability of the laboratory facilities more extensively

ii) Non availability of trained manpower to carry out the investigations

As discussed before non availability of laboratory facilities are more often the result of attitude of the authorities than non availability of the funds. It is often not realized that a reasonably good hematology laboratory with simple coagulation testing facility may not cost more than USD 50,000 to furnish such a laboratory (Equivalent of properly treating a severe hemophilia patient for one year in the western country) and the benefits not only comes for hemophilia patients only but also to all the patients with common hemoglobinopathy, day to day management of serious medical, surgical, gynecological and pediatric patients.

Similarly a simple molecular biology laboratory with one PCR machine, one simple gel viewing apparatus and other ancillaries will not cost more than USD 20,000 - 30,000 but the benefits in terms of genetic diagnosis and disease prevention can be enormous (Preventing birth of one severe hemophilia patient will save the exchequer about 1 million dollars if we calculate the treatment of severe hemophilia patient till he is $18-20$ years of age)

\section{TRAINING THE MANPOWER}

Gynecologists and Sonologists can easily be trained for foetal sampling by a short course of training lasting between $10-14$ days. This training can easily be given at tertiary centers where such procedures are regularly done.

For molecular biology training specific to prenatal diagnosis

One of the best way to develop manpower in this area is by process of twining. Our experience show that a technician can be reasonably trained to do RFLP based genetic diagnosis by $4-6$ weeks of training. Under the help of World Federation of Hemophilia twining programme we have already trained quiet a few such people and they are doing an exemplary job.

For more intensive expertise one of the best wasy is to have students for Ph.D programme. A student in a less developed area can take this topic as his/her Ph.D thesis and through twining programme can successfully start the full fledged laboratory for prenatal diagnosis in his areas. Our center used this method and the techniques learned in Prof. Mannucci's laboratory in Milan has been successfully utilized here.

\section{COUNSELLING}

Genetic counselling is an integral part of prenatal diagnosis , carrier detection or for any other genetic diagnosis. Genetic disorders often causes inappropriate levels of anxiety , feeling of guilt and in some societies stigmalisation.

Females carrying the affected genes are at particular disadvantage.In some societies there could be special problems in ares of Insurance benefits or there could be particular difficulty in getting jobs or getting married. Continuing information, communication and education of the society in a well organized manner is needed to reduce the taboo around genetic disorders. But at the level of Individual patient and families prediagnostic counseling allows patient to understand the disease, provides them with autonomy and requisite knowledge to take proper control of their life and help them to make informed decision as to whether the couple will progress to do the prenatal diagnosis and what they are going to do if the results of prenatal diagnosis is not favourable. Post diagnostic counselling is also extremely useful and should be a must for this kind of disorder as this allows the couple to voice their unresolved questions and inner conflicts which might have arose following prediagnosis counseling. Several short courses on genetic counseling is now available in this country and we found that after training many parents of haemophilia patients became good counselors.

\section{CONCLUSION}

As we control infectious diseases, control early infant mortality, genetic diseases are going to be important health problem. In developing countries hemoglobinopathies al- 
ready pose substantial health problem and couples with this common congenital bleeding disorders like severe hemophilia contribute low volume high cost disease which the developing countries needs to prevent. If we target highest possible level of laboratory efficiency to start with we may never reach there but starting from phenotypic diagnosis, though RFLP and finally mutation detection capability should be aimed for such prevention. Hemoglobinopathies should give the primary impetus to develop more extensive network of simple molecular biology laboratory and hemoglobinopathy detection laboratory coupled with this an elementary coagulation lab will go a long way notably for diagnosis and prevention of hemophilia and it will improve the general level of health care, at least if we target it upto district hsospital in coming decade.

\section{CONTRIBUTIONS}

Kanjaksha Ghosh has planned and written the paper, Kinjalka Ghosh has collected the references, Shrimati Shetty has given the technical inputs and was involved in doing prenatal diagnosis in Haemophilia in more than 500 families at this Institute.

\section{ACKNOWLEDGEMENTS}

Indian Council of Medical Research for supporting Prenatal diagnosis Programme.Dr Shrimati Shetty had her training in carrier detection and prenatal Diagnosis of haemophilia at Prof PM Mannucci's laboratory in Milan,Italy; almost a decade back under the fellowship programme of Haemophilia Federation of India(HFI). Authors KG \& SS gratefully acknowledge the contribution of HFI \& Prof Mannucci in helping NIIH to set up one of the leading Prenatal Diagnosis / carrier detection Centre for Haemophilia In India.

\section{ABBREVIATIONS}

$$
\begin{aligned}
& \text { APTT }=\text { Activated Partial Thromboplastin Time } \\
& \text { PT } \\
& \text { TT } \\
& \text { STR }
\end{aligned}
$$

\section{REFERENCES}

[1] Chandy M. Economics of Haemophilia Care in The Developing World in "Comprehensive Haemophilia care in developing countries". Eds. Sohail MT \& Heijnon L, Ferozsons, Karachi Pakistan, 2001; 1134-39.
[2] Ghosh K, Nair AP, Jijina F, Madkaikar H, Shetty S, Mohanty D. Intracranial haemorrhage in severe haemophilia: prevalence and outcome in s developing country. Haemophilia 2005; 11: 459-62.

[3] http: // plan-international .org/Where we wwork/asia / 5o India / via $=$ text only accessed 27.03.07.

[4] Ghosh K. Management of haemophilia and its complications in developing countries. Clin Lab Haematol 2004; 26: 243-51.

[5] Peake IR, Lillicrap DP, Boulyjenkev E, et al. Report of s joint WHO / WFH meeting on the control of haemophilia carrier detection and antenatal diagnosis. Blood Coagul Fibrinolysis 1993; 4: 313-46.

[6] Shetty S, Ghosh K, Pathare S, Mohanty D. Carrier detection in haemophilia S families: Comparison of conventional coagulation parameters with DNA polymorphism analysis - first report from India. Haemophilia 1999; 5: 243-46.

[7] Elias S, Simpson JL: Amniocentesis in Milumsky A Ed. Genetic disorders and the fetus. $2^{\text {nd }}$ ed. Plenum Press. New York 1986; 4448.

[8] Park D, Genomic DNA isolation from different biological materials. Methods Mol Biol 2007; 353: 3-14.

[9] Shetty S, Ghosh K. Robustness of factor assays follow up cordocentesis in prenatal diagnosis of haemophilia and other bleeding disorders. Haemophilia 2007; 13, 172-177.

[10] Choudhary MR, Tiwari H, Kabra H, B Menon PS. Prenatal diagnosis of using haemophilia A using factor VIII gene polymorphisms Indian experience. Ann Hematol 2003; 427-30.

[11] Ranjan R, Biswas S, Kannan M, Meena A, Deka D, Saxena R. Prenatal diagnosis of haemophilia A by chorionic villus sampling and cordocentesis. All India institute of Medical Sciences experience. Vox Sang 2007; 92: 79-84.

[12] Pandey GS, Phadke SR, Mittal B. Carrier analysis and prenatal diagnosis of haemophilia A in north India. Int J Mol Med 2002; 10: 661-4.

[13] Shetty S, Ghosh K, Jijina F. First Trimestor prenatal diagnosis in haemophilia A and B families . 10 years experience from a center in India. Prenat Diag 2006; 26: 1015-17.

[14] Forestier F, Daffos F, Sole Y, Rainant M. Prenatal diagnosis of haemophilia by fetal blood sampling under ultrasound guidance. Haemostasis 1986; 16: 346-51.

[15] Aseev M, Surin V, Baboev K, et al. Allele frequencies and molecular diagnosis in haemophilia A\& B patients from Russia and from some Asian republics of formor USSR. Prenat. Diag. 1994; 14: 513-22.

[16] Shetty S, Ghosh K, Mohanty D. ST 14 (D X S 52) VNTR polymorphism in the Indian population and its application in carrier detection and prenatal diagnosis of haemophilia A families. Haematologia (Budap) 2000; 36: 203-7.

[17] Shetty S, Ghosh K, Pathare A, Colah R, Badakare S, Mohanty D. Factor VIII and IX gene polymorphism and carrier analysis in Indian population. Am J Haematol 1997; 54: 271-75.

[18] Jenknis PV, Collins PW, Goldman E, et al. Analysis of intron 22 enversion of factor VIII gene in severe haemophilia A: implication for genetic counseling. Blood 1994; 84: 2197-201.

[19] Bagnall RD, Waseem N, Green PM, Gianelli F. Recurrent inversion breaking introns 1 of the factor VIII gene as s frequent cause of severe haemophilia A. Blood 2002; 99: 168-74.

[20] Shetty S Ghosh K, Mohanty D. Alternate strategies for carrier detection and antenatal diagnosis in haemophilia in developing countires. Ind J Hum Genet 2003; 9: 5-9.

[21] Jayandharan G, Shaji RV, Baidya S, Nair SC, Chandy M, Srivastava A. Identification of factor VIII gene mutations in 101 patients with haemophilia A: mutation analysis by inversion screening and multiplex PCR and CSGE and molecular modelling of 10 novel missense substitutions. Haemophilia 2005; 11: 481-91.

[22] Mota L, Ghosh K, Shetty S. Second trimester antenatal diagnosis in rare coagulation factor deficiencies. J Pediatr Haematol Oncol 2007; 29: 137-39. 